\title{
In Vivo and In Situ Detection of Macromolecular Free Radicals Using Immuno-Spin Trapping and Molecular Magnetic Resonance Imaging
}

Rheal A. Towner and Nataliya Smith

\begin{abstract}
Significance: In vivo free radical imaging in preclinical models of disease has become a reality. Free radicals have traditionally been characterized by electron spin resonance (ESR) or electron paramagnetic resonance (EPR) spectroscopy coupled with spin trapping. The disadvantage of the ESR/EPR approach is that spin adducts are short-lived due to biological reductive and/or oxidative processes. Immuno-spin trapping (IST) involves the use of an antibody that recognizes macromolecular 5,5-dimethyl-pyrroline- $N$ oxide (DMPO) spin adducts (anti-DMPO antibody), regardless of the oxidative/reductive state of trapped radical adducts.

Recent Advances: The IST approach has been extended to an in vivo application that combines IST with molecular magnetic resonance imaging (mMRI). This combined IST-mMRI approach involves the use of a spin-trapping agent, DMPO, to trap free radicals in disease models, and administration of an mMRI probe, an anti-DMPO probe, which combines an antibody against DMPO-radical adducts and an MRI contrast agent, resulting in targeted free radical adduct detection.

Critical Issues: The combined IST-mMRI approach has been used in several rodent disease models, including diabetes, amyotrophic lateral sclerosis (ALS), gliomas, and septic encephalopathy. The advantage of this approach is that heterogeneous levels of trapped free radicals can be detected directly in vivo and in situ to pin point where free radicals are formed in different tissues.

Future Directions: The approach can also be used to assess therapeutic agents that are either free radical scavengers or generate free radicals. Smaller probe constructs and radical identification approaches are being considered. The focus of this review is on the different applications that have been studied, advantages and limitations, and future directions. Antioxid. Redox Signal. 28, 1404-1415.
\end{abstract}

Keywords: immuno-spin trapping, targeted free radical imaging, diabetes, amyotrophic lateral sclerosis, glioma, septic encephalopathy

\section{Introduction}

Overview

$\mathbf{T}$ HE FOCUS OF this review is on in vivo and in situ immunospin trapping (IST)-molecular magnetic resonance imaging (mMRI) applications in different experimental oxidative stress-associated animal disease models that have been studied, advantages and limitations of the technique, and future directions in further applications, improvements on the methodology that can be made, and subsequent free radical identification approaches.

The idea initially stemmed from combining the in vitrolex vivo IST technique developed by Mason (37-40) with in vivo imaging so that heterogeneous and tissue-specific levels of free radicals could be visualized in experimental animal disease models. To visualize free radicals in biological systems (e.g., cells and tissues), Mason et al. developed an anti-5,5-dimethylpyrroline- $N$-oxide (DMPO) antibody that could trap radicals

Advanced Magnetic Resonance Center, Oklahoma Medical Research Foundation, Oklahoma City, Oklahoma. 

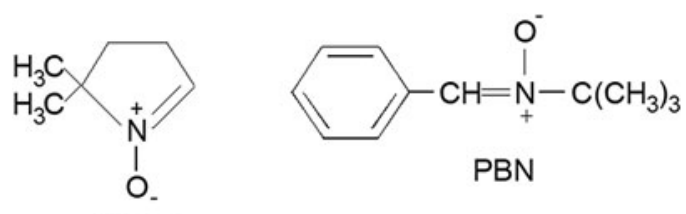

DMPO

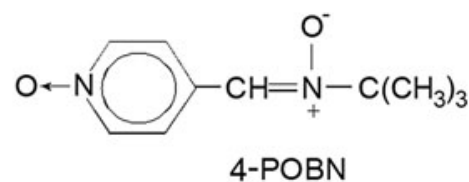

FIG. 1. Common biological spin trapping agents.

$(16,17,31,39,40,44)$, and then be visualized by a fluorescence tag. To visualize the DMPO-trapped radicals in vivo, our group decided to conjugate the anti-DMPO antibody to an MRI contrast agent construct so that DMPO-trapped radicals could be observed spatially either in two or three dimensions with a preclinical MRI system (10, 54-58). High-field MRI was considered due to the superior image resolution (e.g., $\sim 50 \times 50 \mu \mathrm{m}^{2}$ for mice and $\sim 100 \times 100 \mu \mathrm{m}^{2}$ for rats) of these imaging systems.

A brief introduction discussing the use of spin trapping with electron spin resonance (ESR) or electron paramagnetic resonance (EPR), the concept of IST, the combined IST and mMRI approach (focus of this review), alternative approaches by other investigators to detect free radicals in vivo and in situ, and the role of reactive oxygen and nitrogen species (RONS) in various diseases, is presented hereunder.

\section{Spin trapping and ESR/EPR spectroscopy}

For more than half a century, free radicals were characterized by ESR or EPR spectroscopy coupled with spin trapping. Nitrone spin traps ( $N$-oxides of imines), such as $\alpha$-phenyl-tertbutyl nitrone, DMPO, or $\alpha$ (4-pyridyl-1-oxide)- $N$-tert-butyl nitrone (see Fig. 1 for chemical structures), are the most commonly used for biological systems $(1,9,11,12,19,26-28$, $41,53,60)$, and have been administered in vivo in various preclinical disease models $(5,6,37,38)$ for several decades.

The disadvantage of the ESR/EPR approach is that the spin adducts (spin trapping agent-free radical adducts or aminoxyls) are short-lived due to reductive and/or oxidative processes in biological systems $(26,53)$ (Fig. 2).

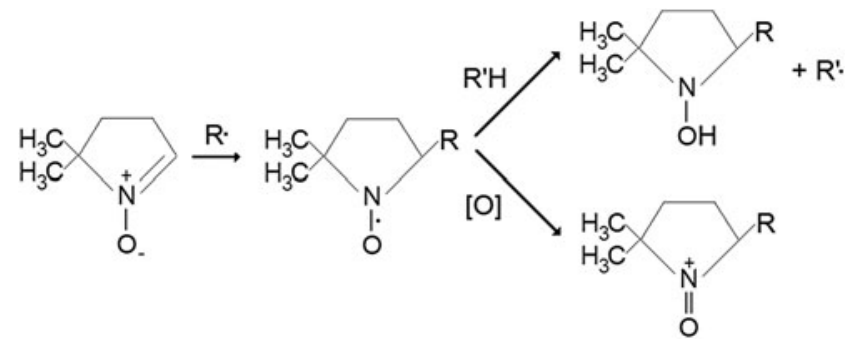

FIG. 2. Spin trapping agents (nitrones) can be used to trap free radical compounds $\left(\mathbf{R}^{\bullet}\right)$ to form a spin adduct (nitroxide or aminoxyl), which are detected by EPR spectroscopy. In biological systems, spin adducts can be either reduced $\left(\mathrm{R}^{\prime} \mathrm{H}\right)$ or oxidized $([\mathrm{O}])$, which are EPR silent. EPR, electron paramagnetic resonance.

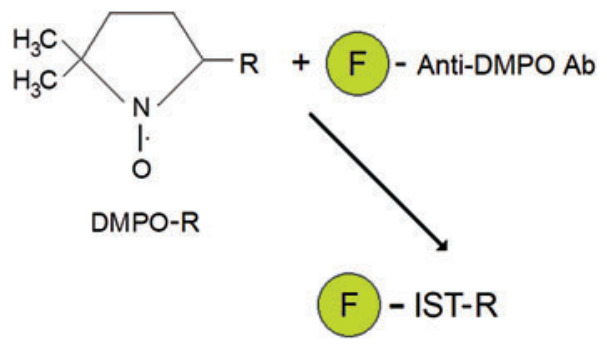

FIG. 3. IST approach. IST, immuno-spin trapping. To see this illustration in color, the reader is referred to the web version of this article at www.liebertpub.com/ars

\section{Immuno-spin trapping}

Mason et al. developed an antibody that recognizes macromolecular DMPO spin adducts, regardless of the oxidative/ reductive state of the trapped radical adducts, and called the methodology IST $(16,17,31,39,40,44)$ (see Fig. 3 for an illustrative description), which has been applied in $>80$ publications. The anti-DMPO antibody is attached to a fluorescent dye, allowing the in vitro or ex vivo detection of trapped DMPO-radical adducts, either as the free radical (aminoxyl), reduced, or oxidized products, with fluorescence microscopy, regardless of whether they are EPR detectable or not.

\section{Combined IST and mMRI detection \\ of in vivo and in situ free radicals}

Towner et al. extended the fluorescence in vitrolex vivo approach to an in vivo approach that involves the use of IST in conjunction with mMRI, currently published in six publications $(10,54-58)$. This involves the use of a spin-trapping agent, DMPO, which is used to trap free radicals in a disease model, and administration of an mMRI probe, called an antiDMPO probe (Fig. 4), which combines an antibody against DMPO-radical adducts and an MRI contrast agent, resulting in targeted free radical adduct mMRI (see Fig. 5 for methodology scheme). The contrast agent used in the Towner approach includes an albumin-gadolinium diethylene tri-

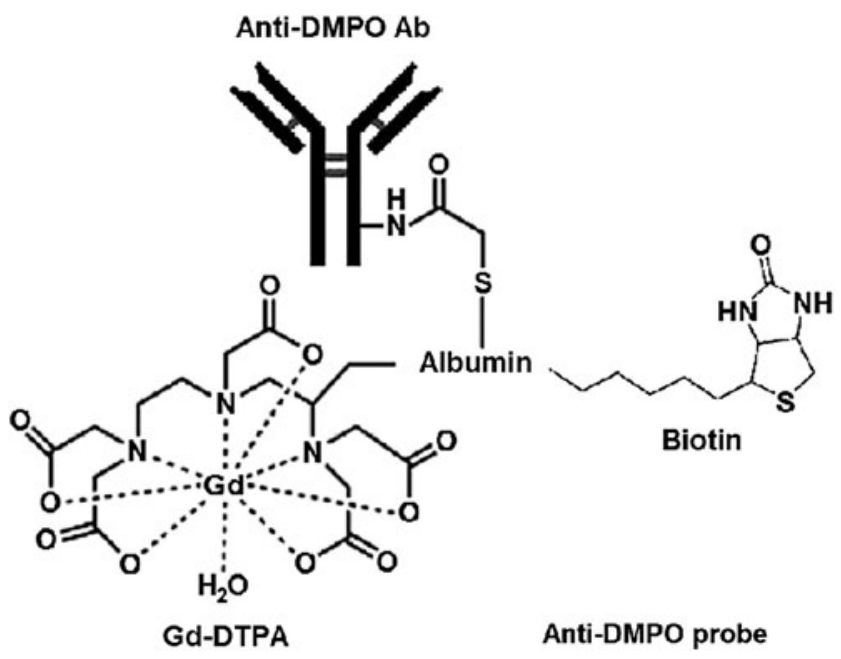

FIG. 4. Illustration of the anti-DMPO probe. Modified from Gomez-Mejiba et al. (17). anti-DMPO probe, antiDMPO-adduct antibodyalbumin-Gd-DTPA-biotin; DMPO, 5,5-dimethylpyrroline-N-oxide. 


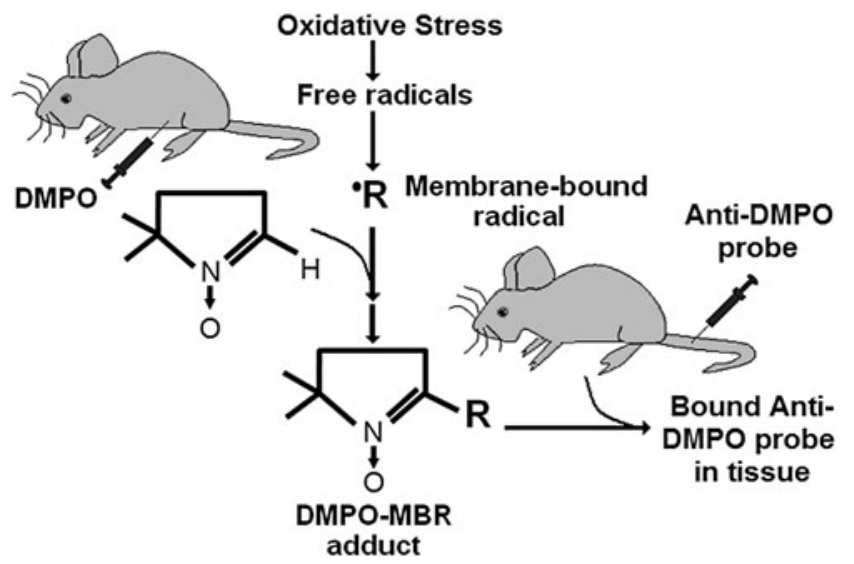

FIG. 5. Combined IST and free radical-targeted mMRI approach. Initially mice are administered DMPO (i.p.) to trap free radicals. Any cell membrane-bound radicals (e.g., oxidized proteins or lipids) can then be detected with the anti-DMPO probe (administered via a tail-vein catheter). Modified from Towner et al. (57). i.p., intraperitoneal; mMRI, molecular magnetic resonance imaging.

amine penta-acetic acid (Gd-DTPA)-biotin construct, where the anti-DMPO antibody is covalently linked to the cysteine residues of albumin, forming an anti-DMPO adduct antibodyalbumin-Gd-DTPA-biotin entity. The Gd-DTPA moiety acts as the MRI signaling component, which will increase MRI signal intensity (SI) in a $\mathrm{T}_{1}$-weighted morphological MRI sequence, and decrease $T_{1}$ relaxation in a $T_{1}$ map image. Both of these parameters, MRI SI or $\mathrm{T}_{1}$ relaxation, can be used to assess the presence of the anti-DMPO probe. The biotin moiety can be used for ex vivo validation of the presence of the anti-DMPO probe in tissues, by using streptavidin fluorescence (e.g., $\mathrm{Cy} 3)$ or streptavidin-horse radish peroxidase (HRP) to tag the biotin in the anti-DMPO probe.

\section{Other approaches used to detect in vivo and in situ free radicals in animal models and cells}

Recent studies by Berkowitz et al. have used quenchassisted (Quest) 1/T $\mathrm{T}_{1}$ MRI to measure oxidative stress changes in rodent models $(3,4)$. Quest MRI detected pathologic free radical production in manganese superoxide dismutase $(M n S O D)$ knockout mouse retinas with laminar resolution in vivo, where, in particular, dark-adapted retinal pigment epithelium-specific MnSOD knockout mice had elevated $1 / T_{1}$ values in the outer retina than relevant controls (4) The Quest MRI technique was also used to report on high levels of free radicals in the hippocampus region in mouse models for neurological diseases such as Alzheimer's disease and Angelman syndrome (3). However, it should be noted that paramagnetic oxygen $(3,4,51)$ and hydrogen peroxide $(3$, 51) can also provide a dominant $1 / \mathrm{T}_{1}$ contrast effect, which could complicate the interpretation of the presence of free radicals. In addition, temperature and $\mathrm{pH}$ can also influence the rates of proton exchange that will also affect $1 / \mathrm{T}_{1}$ contrast $(30,50)$. Nonetheless, Quest MRI is an interesting approach that provides information on total free radical burden, somewhat similar to the combined IST and mMRI approach, but without the use of an MRI contrast agent $(3,4)$.
Endogenous reactive oxygen species (ROS) contrast MRI was also recently used by Tain et al. to detect ROS (measured as a reduction in $\mathrm{T}_{1}$ ) in rotenone-treated mouse brains (51). Another study by Eto et al. used in vivo dynamic nuclear polarization MRI (DNP-MRI) with nitroxyl radicals (carbamoyl-2,2,5,5-tetramethylpyrrolidine-1-oxyl [PROXYL] [cell permeable] and carboxy-PROXYL [cell impermeable]) to assess the redox status (measured as an increase in image intensity) in the skeletal muscle of mice that had an acute local inflammation (induced with intramuscular injection of bupivacaine) (14). The signal decay of carbamoylPROXYL in bupivacaine-exposed mice was confirmed by in vivo L-band EPR spectroscopy (14). The nitroxyl radical probes are paramagnetic, which broaden the MRI signal, bind free radicals, and thus results in an increase in MRI SI (29). Another group used DNP-MRI to visualize endogenous free radical intermediates of flavin mononucleotide-hydrogen (FMNH) and flavin adenine dinucleotide-hydrogen (FADH) in vitro (22), which could potentially be detected in vivo in the future. DNP-MRI, also used for proton electron doubleresonance imaging or overhauser enhanced magnetic resonance imaging (OMRI), is a relatively new imaging approach for detecting free radical species in vivo $(23,59)$. For instance, DNP studies can be carried out using ${ }^{15} \mathrm{~N}$-labeled carbamoylPROXYL in biological systems (32). DNP-MR images are derived from the intensity-enhanced images of solvent protons (e.g., water protons for FMNH, FADH, and carbamoylPROXYL; and nonaqueous hydrocarbon protons for $\mathrm{CoQ}_{10} \mathrm{H}$, and vitamins $\mathrm{E}$ and $\mathrm{K}_{1}$ radicals) (59). Yamamoto et al. recently developed a combined positron emission tomography/OMRI system to detect radionucleotide and nitroxyl radical probes for small animal imaging (61).

It is well known that intensity-based fluorescent methods (particularly $2^{\prime}, 7^{\prime}$-dicholorofluorescin) for ROS (includes the nonradical hydrogen peroxide) detection/quantification are sensitive and readily used; however, these agents lack the specificity for ROS or reactive nitrogen species, and often produce artifacts resulting in false-positive signals $(7,46)$. An interesting recent study by Liu et al. used a new fluorescent probe, MPT-Cy2, which can be used to detect endogenous in vivo hydroxyl radicals in cells and zebrafish (35). MPT-Cy2 becomes a fluorescent product, OMPT-Cy2, when it binds hydroxyl radicals (35). In a similar manner, Hu et al. reported on a fluorescent probe, HKSOX-1, for the imaging and detection of endogenous in vivo superoxide in cells and zebrafish embryos (20). A fluorescence probe, o-phenylene diaminePhe-Phe-OH, has also been developed for the detection of nitric oxide (NO), and used in living cells (36). Another fluorescence probe, LyNP-NO, was used to detect NO in C6 glioma cells (18). It was also found that single-walled carbon nanotubes have fluorescent properties and have been used to detect in vivo levels of $\mathrm{NO}$, which quenches the fluorescence signal, in mice (25). However, for most of the fluorescence probes, in vivo applications will be limited to a depth-of-penetration detection of the fluorescence signal and may only be applicable to small animal models.

Another group, Li et al., used a near infrared (NIR) lightexcited luminescence resonance energy transfer-based nanoprobe for in vivo detection of hydroxyl radicals (34). NIR fluorescence probes (Hcy-Mito and Hcy-Biot) were also recently used for the in situ detection of superoxide anion and hydrogen polysulfides in living cells and in mouse tumor 
models (21). Also, a phosphinate-based NIR fluorescence probe, CyR, was recently also used to detect superoxide radical anion in vivo within zebrafish (62). NIR fluorescent single-walled carbon nanotubes have also been used to detect in vivo NO levels in mice (24). It should be pointed out, however, that there is ESR spectroscopy evidence for in vivo formation of free radicals in the tissues (lungs, heart, and liver) of mice exposed to single-walled carbon nanotubes with no oxidative stress (48).

Rayner et al. used a reversible profluorescent probe containing a redox-sensitive nitroxide moiety (methyl ester tetraethylrhodamine nitroxide) for the in vivo detection of retinal oxidative status within rat retina after acute ischemiareperfusion injury (45). An interesting approach using nitrone-functionalized gold nanoparticles (Au@EMPO, $\mathrm{EMPO}=2$-(ethoxycarbonyl)-2-methyl-3,4-dihydr-2H-pyrrole 1-oxide) to trap hydroxyl radicals was demonstrated by Du et al. (13) and may be potentially important for preclinical in vivo applications in combination with microcomputed tomography.

Lastly, ESR imaging has also been used to detect in vivo levels of endogenous free radicals with probes that form nitroxides when they react with ROS. For instance, Togashi et al. used an in vivo ESR imaging system to visualize hepatic oxidative stress in an acute immune-mediated hepatic injury model in mice using 1-acetoxy-3-carbamoyl-2,2,5,5tetramethylpyrroline, ACP, as a probe that produces an ESR-detectable nitroxide upon reaction with any ROS (52).

\section{Free radicals in various diseases}

RONS lead to structural and functional modifications of cellular proteins and lipids, resulting in cellular dysfunction, such as impaired energy metabolism, altered cell signaling and cell cycle control, impaired cell transport processes and dysfunctional biological activities, immune activation, and inflammation (42). RONS can be involved in several disease processes as causative agents or result as an effect of the pathogenesis. It is well known that free radicals play a role in the pathogenesis associated with various diseases such as diabetes, septic encephalopathy, neurodegenerative diseases, and cancers, to mention a few.

Nutritional stress, which for instance may result from excessive high-fat and/or carbohydrates, can promote oxidative stress, subsequently forming lipid peroxidation products, protein carbonylation, and decreased antioxidant levels (42). Chronic oxidative stress and inflammation, both associated with obesity, can lead to insulin resistance, dysregulated metabolic pathways, diabetes, and cardiovascular diseases, via impaired signaling and metabolism that result in insulin secretion dysfunction, insulin action, and immune responses (42). In type 1 diabetes mellitus, RONS released from phagocytes may damage adjacent cells, which can lead to excessive inflammation and an autoimmune attack against pancreatic islet $\beta$-cells, and contribute to a rapid progression of pathogenesis (42). Immune system-associated enzymes (such as nicotinamide adenine dinucleotide phosphate [NADPH]-oxidase) can trigger the formation of ROS (42). Excessive glucose and lipid levels, endocrine factors, and numerous proinflammatory cytokines are known to activate NADPH oxidase (42). Proinflammatory cytokines can also upregulate nitric oxide synthase 2 , NOS2, producing excessive $\mathrm{NO}$, which can subsequently lead to the formation of perox- ynitrite, and lead to further oxidative stress (42). In Type 2 diabetes mellitus, excessive RONS production from chronic hyperglycemia increases oxidative stress in tissues that exacerbate the disease, such as pancreatic islets, muscle, adipose, and hepatic, as well as influences secondary diabetic complications, including nephropathy, vascular disease, and retinopathy, leading to oxidized lipids and proteins $(15,42)$.

Oxidative stress has been proposed as a contributory factor in the pathogenesis of several neurodegenerative diseases (43). For instance, in familial amyotrophic lateral sclerosis (ALS) (accounting for $5-10 \%$ of ALS cases), there is a mutation in superoxide dismutase 1 (SOD1) that results in dysfunctional superoxide radical clearance, leading to increased oxidative stress (43). NADPH oxidases have emerged as possible drug targets for the treatment of neurodegeneration, due to their role in generating oxidants and also regulating microglia activation (49).

Sepsis-associated encephalopathy pathophysiology is still poorly understood, but a number of mechanisms-of-action (MOA) have been proposed, including mitochondrial and vascular dysfunction, oxidative damage, neurotransmission disturbances, inflammation, and cellular death $(2,8)$. Oxidative stress is a central MOA of acute brain damage (8). Systemic inflammation induces mitochondrial dysfunction, which is involved in both apoptotic and necrotic cell death pathways, and increased glucose uptake by brain tissues, which results in the diversion of glucose to the pentose phosphate pathway that may contribute to oxidative stress by producing excessive superoxide radicals via NADPH oxidase $(2,8)$. In addition, microglia activation results in the secretion of NO, ROS, and matrix metalloproteinases that can all contribute to blood-brain barrier (BBB) and neuronal damage (8). Regarding brain dysfunction in sepsis, it is thought that RONS, generated during a systemic inflammatory response, triggers lipid peroxidation due to a decreased antioxidant activity (2). Free radical-induced structural membrane damage also induces neuroinflammation (2). The formation of excessive superoxide radicals also depletes ambient NO in the cerebrovascular bed, resulting in the formation of peroxynitrite, which irreversibly inhibits the mitochondrial electron transport chain, resulting in an increase in mitochondrial release of free radicals, and leads to mitochondrial dysfunction and neuronal bioenergetics failure (2). In addition, free radicals trigger apoptosis via altering intracellular calcium homeostasis in brain regions such as the cerebral cortex and hippocampus, further exacerbating local inflammatory responses further (2).

In cancer cells, RONS accumulation can result in damaging DNA, directly through an increase in cellular mutations and/or increase in oncogenic phenotypes, or indirectly by acting as secondary messengers intracellular signaling cascades (47). It is thought that impaired cellular repair mechanisms induced by RONS oxidative stress on DNA can lead to cell injury and subsequently to genomic instability, mutagenesis, and tumorigenesis (47). It is also known that ROS can promote cell proliferation, activating growth-related signaling pathways (47). ROS may be involved in the multistep oncogenesis process at various different phases related to tumor initiation and progression, ROS-related mechanisms during tumor promotion, maintenance of the transformed state through extracellular superoxide radical formation by NADPH oxidase 1, and resistance to oxidative stress signals through membrane-associated catalase expression (47). 


\section{In Vivo and In Situ Targeted Free Radical Detection in Various Disease Models}

The combined IST-mMRI approach has been used in several in vivo disease models, including multitissue assessment in diabetic mice (57) (Fig. 6) with further assessment of cardiomyopathy (55) (Fig. 7), and in neurological applications, such as rodent models for ALS (58) (Fig. 8), gliomas (56), and septic encephalopathy (54) (Fig. 9).

\section{Streptozotocin-induced diabetes mouse model}

For the diabetes study, initially all major organs, excluding the heart, such as the lungs, liver, and kidneys, were assessed regarding levels of trapped in vivo and in situ DMPO-radical adducts (57). Figure 6 depicts the data obtained in the liver of diabetic (streptozotocin [STZ]-induced) and nondiabetic mice (wild-type [WT] mice not administered STZ), and appropriate controls (e.g., diabetic mice given saline rather than DMPO plus the anti-DMPO probe-spin trap control; or a diabetic mouse administered DMPO, but given a nonspecific IgG contrast agent [IgG-albumin-Gd-DTPA-biotin] instead of the anti-DMPO probe). Diabetic mice that were administered DMPO and the anti-DMPO probe had significantly higher levels of the anti-DMPO probe (detected by an in- crease in percentage change in MRI SI) than nondiabetic mice or diabetic mouse controls in their lungs, kidneys, and livers (see Fig. 6A for overall distribution of the anti-DMPO probe in a horizontal image, and Fig. 6B for quantitative liver data). A postcontrast image minus a precontrast image was obtained, and the gray-scale image was false-colored red, and overlaid on top of the morphological image. Nonspecific biodistribution of the anti-DMPO was also found in the stomach, intestines, and bladder (Fig. 6A). From kinetics assessment, it was found that the anti-DMPO probe persisted in certain tissues (e.g., lungs, liver, and kidneys) for $>3 \mathrm{~h}$. Verification of the presence of the anti-DMPO probe in $e x$ vivo tissues was done by using streptavidin-Cy3, which bound to the biotin moiety of the anti-DMPO probe (see Fig. 6C for example in liver tissue). It was also confirmed that DMPO-radical adducts were formed by using a fluorescent anti-DMPO antibody (IST approach) in diabetic (Fig. 6F) and nondiabetic mouse livers. In addition, inducible nitric oxide synthase (iNOS) levels were assessed in the livers of diabetic and nondiabetic mice (see Fig. 6D for immunohistochemistry (IHC) detection of iNOS in a diabetic mouse liver; and Figure $6 \mathrm{E}$ for quantitative levels of iNOS in diabetic and nondiabetic mouse livers) as an additional marker of oxidative stress. This was the first in vivo study to demonstrate that
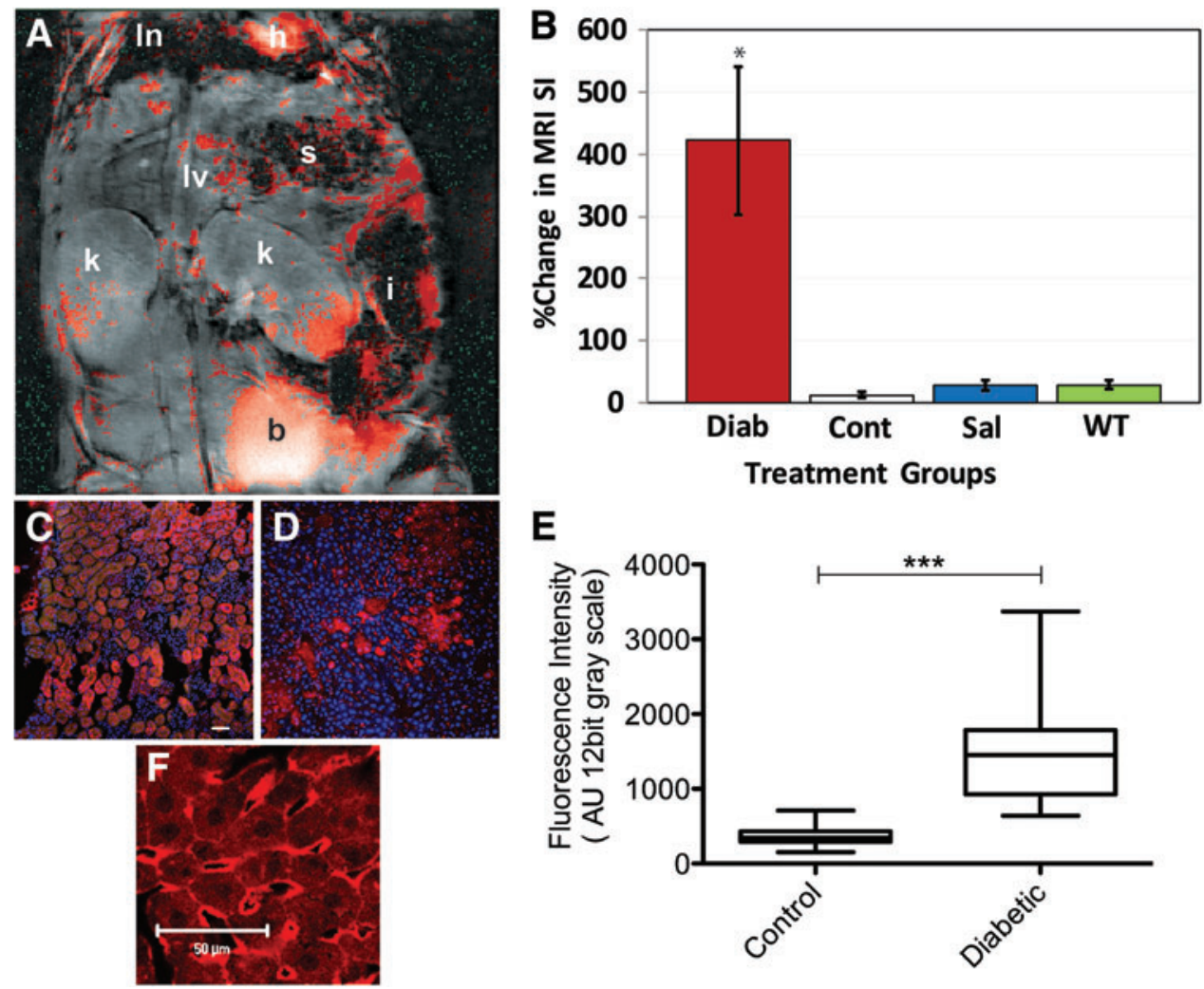

FIG. 6. Combined IST and free radical-targeted mMRI in a STZ-induced mouse diabetic model. (A) In vivo antiDMPO probe distribution map (MRI SI change). Anatomical assignments: ln, lung; h, heart; s, stomach; lv, liver; k, kidney; $\mathrm{i}$, intestine; and b, bladder. (B) Percentage $(\%)$ change in MRI SI in diabetic mice administered DMPO+anti-DMPO probe (Diab), DMPO+a nonspecific IgG contrast agent (Cont), saline+anti-DMPO probe (Sal), and WT nondiabetic mice administered DMPO+anti-DMPO probe. $N=5$ for each group. (C) Stretavidin-Cy3 biotin-tagged ex vivo liver image in diabetic mice. (D) iNOS IHC from a diabetic mouse liver. The scale bar $=50 \mu \mathrm{m}$. (E) iNOS IHC in nondiabetic (control) and diabetic mouse livers. $N=5$ for each. $* p<0.05$, ***p $p 0.001$. (F) Fluorescent-IST of diabetic mouse liver. The scale $\mathrm{bar}=50 \mu \mathrm{m}$. Modified from Towner et al. (57). IHC, immunohistochemistry; iNOS, inducible nitric oxide synthase; SI, signal intensity; STZ, streptozotocin; WT, wild-type. To see this illustration in color, the reader is referred to the web version of this article at www.liebertpub.com/ars 

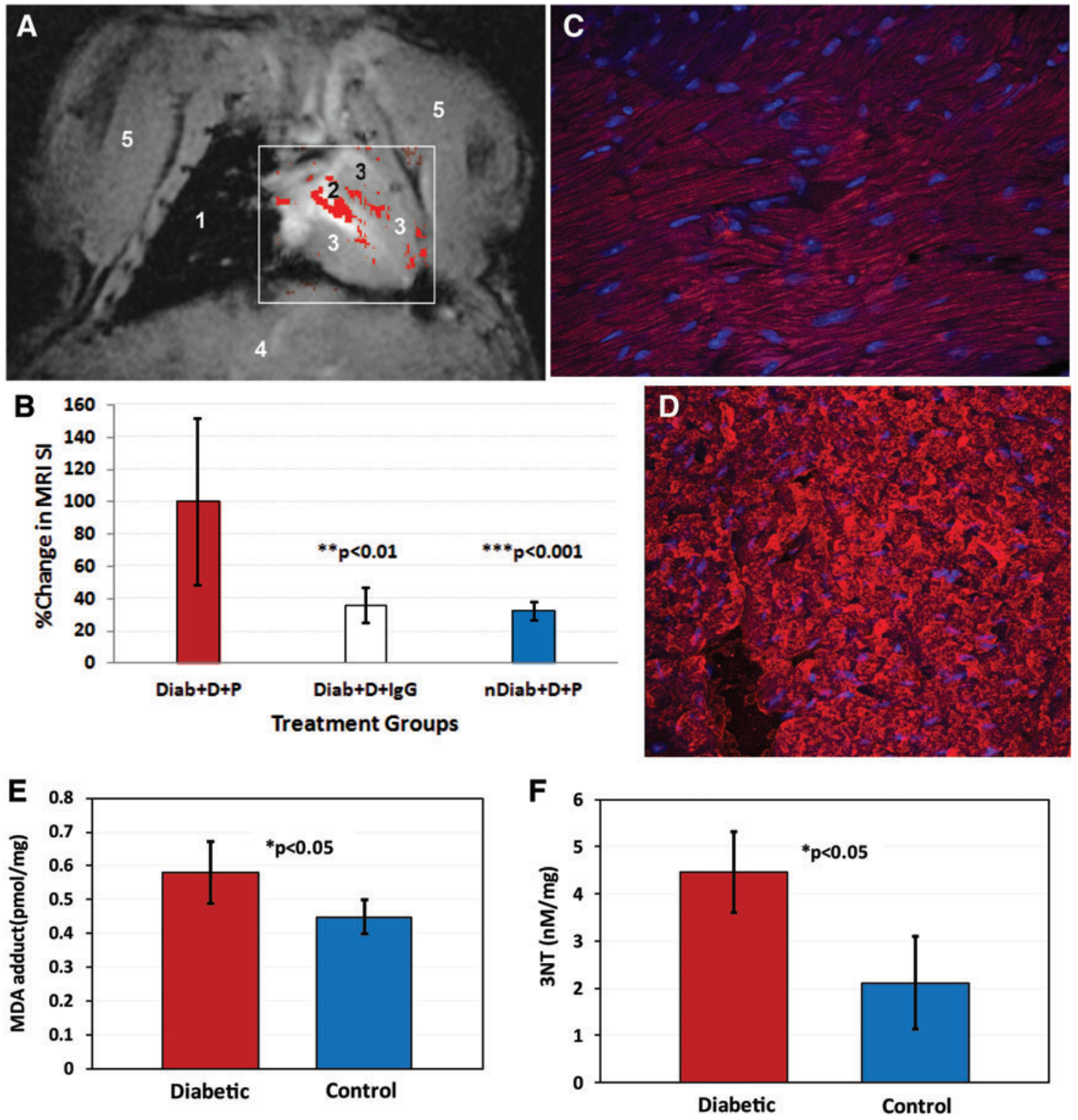

FIG. 7. Combined IST and free radical-targeted mMRI in a STZ-induced mouse diabetic heart. (A) MR image of a mouse heart with an in vivo anti-DMPO probe cardiac map overlay (MRI SI change). Anatomical assignments: 1, lung; 2, left ventricle chamber; 3, cardiac muscle; 4, liver; and 5, thoracic muscle. The inset is the colorized free radical-targeted molecular image overlay on top of the contrast difference image. (B) Percentage (\%) change in MRI SI in diabetic mouse hearts after administered DMPO+anti-DMPO probe (Diab+D+P), DMPO+a nonspecific IgG contrast agent (Diab+D+IgG), and nondiabetic mice administered DMPO+anti-DMPO probe (nDiab+D+P). $N=5$ for each. (C) Stretavidin-Cy3 biotin-tagged ex vivo cardiac image in diabetic mice. (D) Fluorescent-IST diabetic mouse heart. (E) MDA adducts and (F) 3-NT enzyme-linked immunosorbent assays from the hearts of diabetic and nondiabetic mice. $N=5$ for each. Modified from Towner et al. (55). 3-NT, 3-nitrotyrosine; MDA, malondialdehyde. To see this illustration in color, the reader is referred to the web version of this article at www.liebertpub.com/ars

diabetic mice had elevated in situ free radical levels in organs/tissues such as the lungs, liver, and kidneys.

At a later stage, it was found that the cardiac muscle in diabetic mice also retained the anti-DMPO probe (55) (Fig. 7). A morphological MR image of a mouse heart is shown in Figure 7A. The postcontrast minus precontrast image in a diabetic mouse with false coloration is shown in Figure 7A, overlaid on top of a horizontal morphological image of the heart. Significantly higher quantitative levels of the anti-DMPO probe in diabetic (Diab) mice administered DMPO (D) and the anti-DMPO probe $(\mathrm{P})$ were found when compared with diabetic (administered the isotype IgG contrast agent instead of the anti-DMPO probe) and nondiabetic (non-STZ-exposed WT mice administered DMPO and the anti-DMPO probe) controls (Fig. 7B). Confirmation of the presence of the anti-DMPO probe in cardiac muscle of a diabetic mouse is shown in Figure 7C. Verification of the presence of DMPO-radical adducts is shown in Figure 7D, where a fluorescent-labeled anti-DMPO antibody was used. In the diabetic cardiomyopathy study, it was also established that diabetic mice had significantly higher levels of malondialdehyde (MDA) adducts (oxidized lipid marker) (Fig. 7E) 

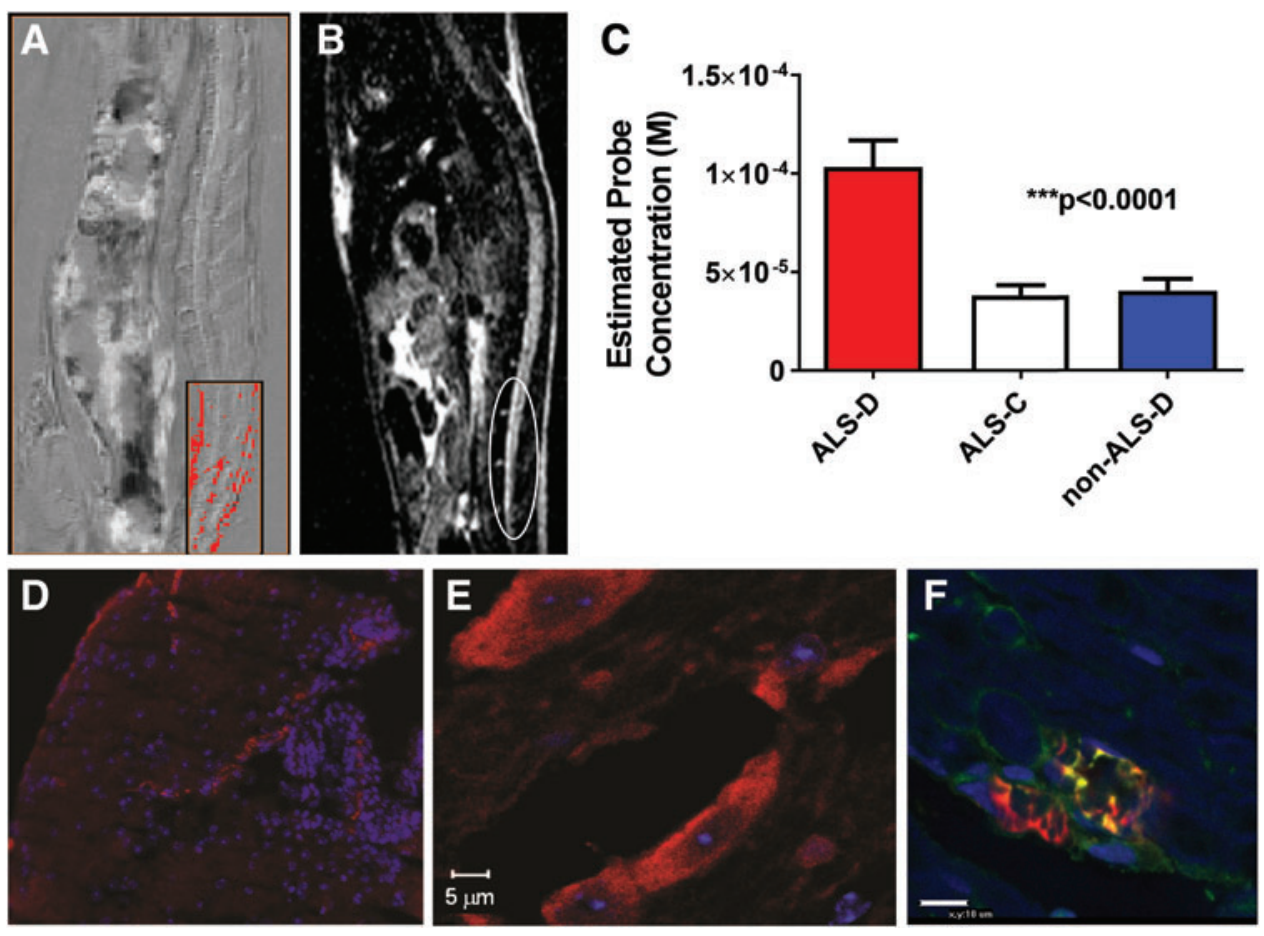

FIG. 8. Combined IST and free radical-targeted mMRI in an ALS mouse model. (A) In vivo anti-DMPO probe map (MRI SI change). The rectangular inset is the colorized free radical-targeted molecular image overlay on top of the contrast difference image. (B) Diffusion-weighted image of an ALS mouse with increased ADC in lumbar region (outlined in the oval region). (C) DMPO probe concentration in ALS mice administered DMPO+anti-DMPO probe (ALS-D), DMPO+nonspecific IgG contrast agent (ALS-C), and WT non-ALS mice administered DMPO+anti-DMPO probe (nonALS-D). $N=5$ for each group. (D) Stretavidin-Cy3 biotin-tagged ex vivo spinal cord in ALS-D. (E) Fluorescent-IST of spinal cord of an ALS mouse. (F) Colocalized DMPO probe (red) and neuronal marker (green) fluorescence image in mouse spinal cord. Modified from Towner et al. (58). ADC, apparent diffusion coefficient; ALS, amyotrophic lateral sclerosis. To see this illustration in color, the reader is referred to the web version of this article at www.liebertpub.com/ars

and 3-nitrotyrosine (3-NT) (oxidized protein marker) (Fig. 7F) in cardiac muscle than nondiabetic mice. This was the first in vivo study to demonstrate increased in situ free radical levels in diabetic cardiomyopathy. The correlation with oxidized lipids and proteins was done, as it is suspected that the combined IST and mMRI approach primarily reports on macromolecular free radicals that are cell membrane bound. Mason et al. have previously reported on the application of IST to trap oxidized proteins $(16,39,44)$. As an increase in both oxidized lipids and proteins was detected in diabetic cardiac muscle via enzyme-linked immunosorbent assay, it is possible that the combined IST and mMRI method detects both oxidized lipids and proteins. Further verification would require a mass spectrometry approach to confirm this assumption.

\section{Transgenic ALS mouse model}

High levels of trapped DMPO-radical adducts were also found in a mouse model for ALS (superoxide dismutase [SOD] mutation) (58) (Fig. 8). As SOD1 plays an important role in superoxide radical anion, $\mathrm{O}_{2}^{-\bullet}$, clearance, the loss of SOD1 can lead to increased levels of free radicals (43). A precontrast minus postcontrast difference sagittal image is shown in Figure 8A for an ALS mouse, with false coloration depicted in the lumbar region of the spinal cord. A diffusionweighted image is shown in Figure 8B, depicting a high SI in the lumbar region of the spinal cord. From $\mathrm{T}_{1}$ relaxation values, the estimated anti-DMPO probe concentration can be obtained, and it was found that ALS mice administered DMPO and the antiDMPO probe (ALS-D) had significantly higher levels of the anti-DMPO probe than ALS (administered the IgG contrast agent instead of the anti-DMPO probe) and non-ALS (administered both DMPO and the anti-DMPO probe) controls (Fig. 8C). Ex vivo detection of the anti-DMPO probe in the lumbar region of the spinal cord of an ALS mouse is shown in Figure 8D, and confirmation of DMPO-radical adducts is depicted in Figure 8E. Figure 8F illustrates that a neuronal marker (NrCAM) (green) colocalizes with the location of the antiDMPO probe (red) in some regions (yellow). This is the first direct in vivo detection of elevated in situ free radicals in the lumbar region of ALS-like mice.

\section{Cecal ligation and puncture mouse model for septic encephalopathy}

Mice with septic encephalopathy (induced by cecal ligation and puncture [CLP]) were also found to have higher levels of trapped DMPO-radical adducts than sham animals (abdominal incision without CLP and sutured) (54) (Fig. 9). Figure 9A depicts an MRI SI difference image (false-colored red) overlaid on top of a morphological image of the brain region of a septic mouse. The distribution of the anti-DMPO probe is dispersed throughout the brain, and was found to be significantly higher in septic mice versus sham animals in the 

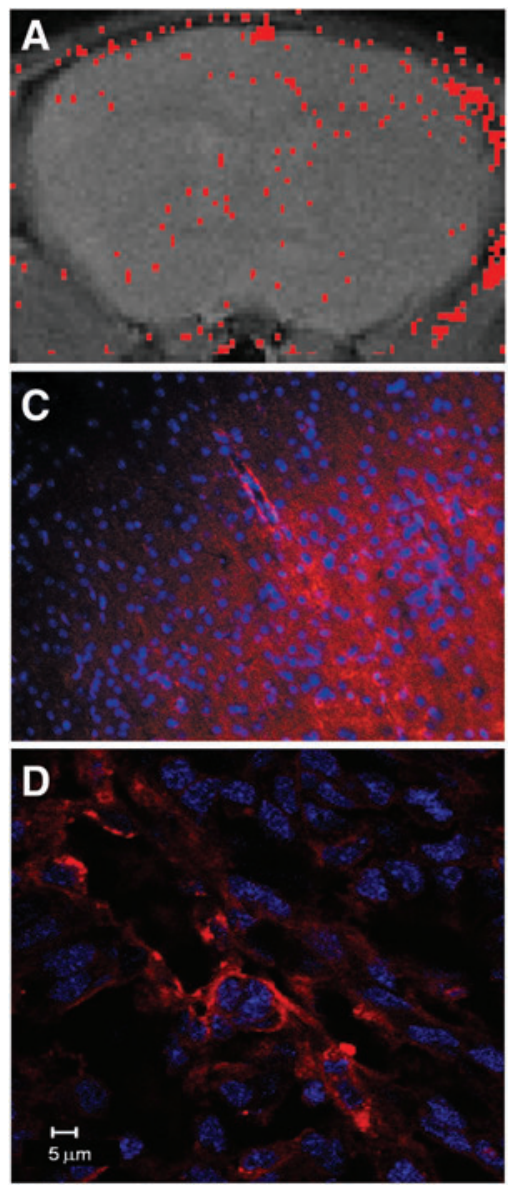

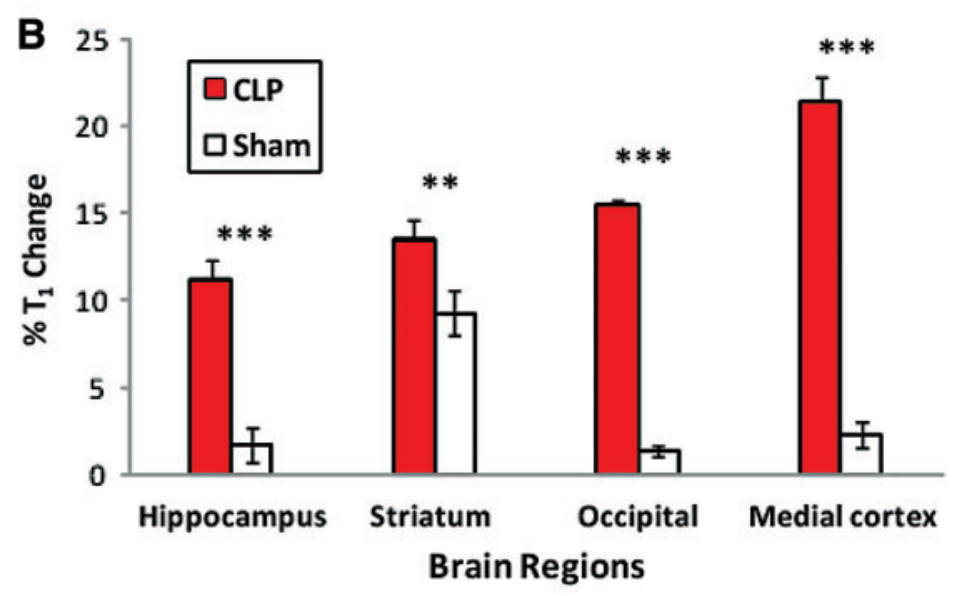

E

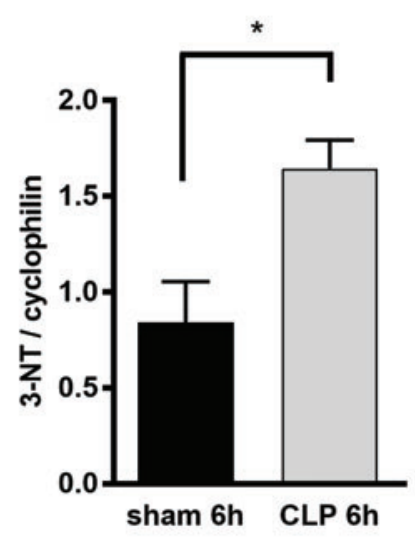

FIG. 9. Combined IST and free radical-targeted mMRI in a septic encephalopathy CLP-induced mouse model. (A) AntiDMPO probe brain map of a septic mouse (based on MRI SI change). (B) Percentage T1 relaxation change in septic and sham mice administered DMPO+anti-DMPO probe in hippocampus, striatum, occipital lobe, and medial cortex. $N=5$ for each. (C) StretavidinCy3 biotin-tagged ex vivo septic brain image. (D) Fluorescent-IST of septic mouse brain. (E) 3-NT levels (Western blots) in brains of septic and nonseptic mice. (F) 4-HNE levels (Western blots) in brains of septic and nonseptic mice. $N=5$ for each. $* p<0.05$, ${ }^{* *} p<0.01,{ }^{* * *} p<0.001$. Modified from Towner et al. (54). 4-HNE, 4-hydroxynonenal; CLP, cecal ligation and puncture. To see this illustration in color, the reader is referred to the web version of this article at www.liebertpub.com/ars

hippocampus, striatum, occipital lobe, and medial cortex regions of the brain (Fig. 9B), as measured by a percentage change (overall decrease in $\mathrm{T}_{1}$ relaxation). Confirmation of the presence of the anti-DMPO in the cortical brain tissue of a septic mouse is shown in Figure 9C, and verification of DMPO-radical adducts in a septic mouse brain is depicted in Figure 9D. Oxidized proteins (measured from Western blots for 3-NT) (Fig. 9E) and oxidized lipids (measured from Western blots for 4-hydroxynonenal [4-HNE]) (Fig. 9F) were found to be significantly higher in septic mice (CLP) after $6 \mathrm{~h}$ than sham controls. This study also indicates that both oxidized lipids and proteins may play a role in the free radicalassociated pathology of ALS. This is the first reported in vivo detection of elevated in situ free radicals in a mouse model for septic encephalopathy.

\section{Confirmation regarding the detection of the anti-DMPO probe in the plasma membrane}

Immunoelectron microscopy (IEM) with gold antibiotin, targeting the biotin moiety of the anti-DMPO probe, was also used to confirm the ex vivo presence of the anti-DMPO probe in the plasma membrane of rat glioma cells following in vivo administration (10) (Fig. 10). The IEM data are confirmation that the combined IST and mMRI approach is detecting macromolecular membrane-bound (both plasma membrane and possibly nuclear membrane) free radicals.

\section{Assessment of the free radical scavenging activity of an anticancer agent in rat gliomas}

The approach can also be used to assess possible therapeutic agents that are either free radical scavengers or generate free radicals. For example, this approach was used to assess the free radical scavenging ability of an anticancer agent, OKlahoma nitrone (OKN-007), in a rat glioma model (10) (Fig. 11). Representative difference images (false-colored red) of an untreated (UT) F98 glioma and an OKN-007-treated F98 glioma, overlaid over appropriate morphological images, are shown in Figure 11A, B, respectively. Quantitative levels of trapped free radical levels (measured from percentage changes in MRI signal intensities) for UT and OKN-007treated F98 gliomas are shown in Figure 11C. Significantly lower levels of 3-NT (Fig. 11F) and MDA (Fig. 11G) were 

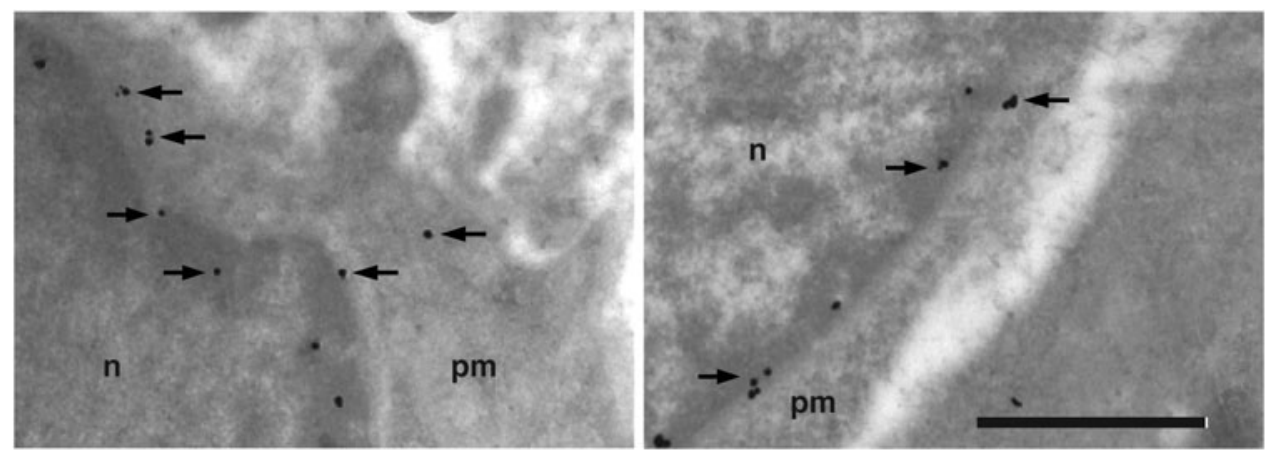

FIG. 10. IEM detection of anti-DMPO probe in plasma membrane/cytoplasm and cell nuclei in F98 rat gliomas. Biotin moiety of anti-DMPO probe was targeted with gold antibiotin. Gold antibiotin colloids were detected within the plasma membrane/cytoplasm (black arrows) and cell nuclei membranes of F98 tumor cells administered the anti-DMPO probe. Scale $b a r=1 \mu \mathrm{m}$. Magnification $=20,000 \times$. Modified from Coutinho de Souza et al. (10). c, cytoplasm; $n$, nucleus; pm, plasma membrane.
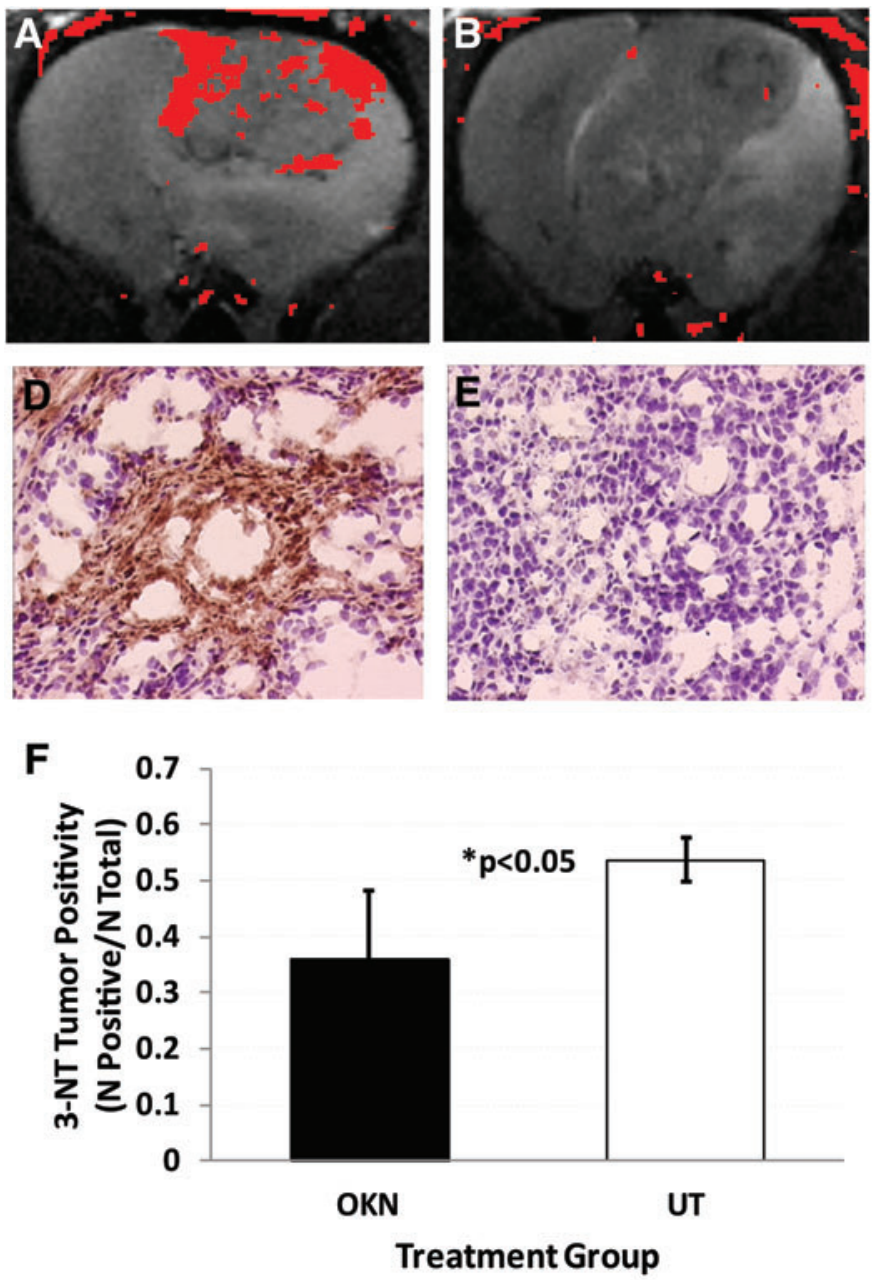
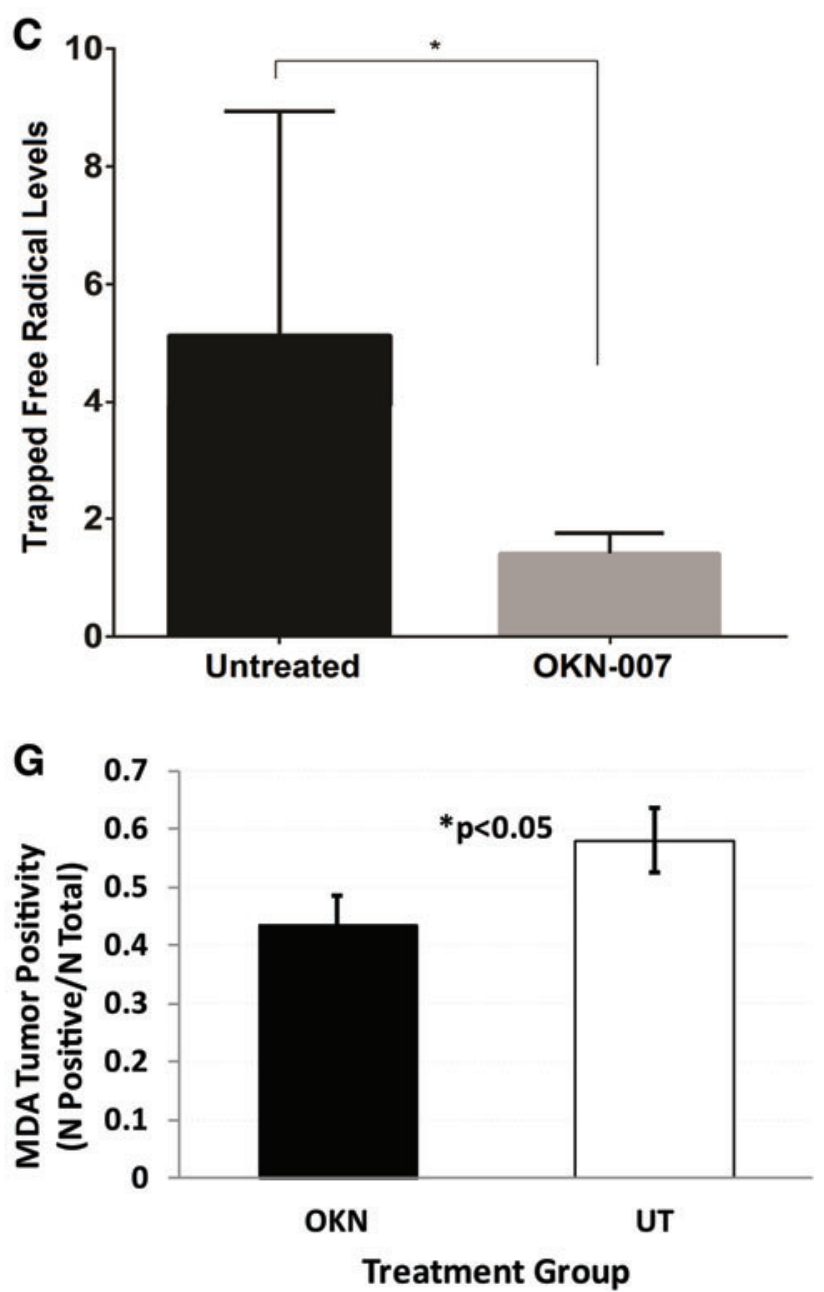

FIG. 11. Combined IST and free radical-targeted mMRI in a F98 rat glioma model. Anti-DMPO probe brain maps of (A) UT and (B) OKN-007-treated rat F98 tumors (MRI SI change). (C) Trapped free radical levels (percentage change in MRI SI) in UT and OKN-007-treated F98 gliomas administered DMPO+anti-DMPO probe. $N=5$ for each group. Stretavidin-HRP biotin-tagged ex vivo F98 gliomas that were (D) UT or (E) OKN-007 treated. (F) 3-NT levels (IHC) in F98 tumors of OKN-007-treated or UT rats. (G) MDA levels (IHC) in F98 tumors of OKN-007-treated or UT rats. $N=5$ for each. Modified from Coutinho de Souza et al. (10). HRP, horse radish peroxidase; OKN-007, OKlahoma nitrone; UT, untreated. To see this illustration in color, the reader is referred to the web version of this article at www.liebertpub.com/ars 
found for F98 gliomas treated with OKN-007 than UT tumors. IHC levels for 3-NT and MDA were quantitated in several $\mathrm{OKN}-007$-treated and UT F98 tumor-bearing rats. These results indicate that $\mathrm{OKN}-007$ acts as a free radical scavenger when used as an anticancer agent. This is the first time in vivo detection of in situ free radicals had been reported for an anticancer agent with free radical scavenging capability. It was previously demonstrated that OKN-007 can significantly increase animal survival and significantly decrease tumor volumes than UT animals. The combined IST and mMRI approach can be taken to assess any therapeutic agents that would either increase or decrease free radical levels in different disease models.

\section{Disadvantages of the Combined IST-mMRI Approach}

Some of the disadvantages with the methodology include limited access to preclinical MRI systems, availability of the anti-DMPO antibody, and further identifying the radical source that is being trapped. For non-neurological studies, this approach can be easily utilized in numerous pathological/ toxicological models. However, for neurological studies, the approach will be limited to whether there is BBB permeability, to allow the anti-DMPO probe, and possibly DMPO, to access the target tissue.

\section{Conclusions}

This review has discussed all of the current studies that have utilized combined IST and mMRI to detect targeted trapped macromolecular DMPO-radical adducts in vivo and in situ within various animal models, wherein oxidative stress plays a major role. It was established for all oxidative stress-associated disease models studied thus far that levels of free radicals were found to be significantly higher in all cases for animals treated with DMPO and the anti-DMPO probe than those of controls, including disease controls (e.g., WT rodents or shams), nonDMPO controls (i.e., administered saline instead of DMPO), and/or mMRI probe controls (i.e., a nonspecific IgG was covalently bound to the albumin of the MRI contrast agent construct instead of the anti-DMPO antibody). An example of assessing an anticancer agent with free radical scavenging activity was also presented. The biotin moiety of the anti-DMPO probe also allowed ex vivo validation of the presence of trapped DMPO macromolecular adducts in various tissues. IST was also used in all cases to confirm the presence of trapped free radicals with fluorescence or optical (e.g., HRP) microscopy. Finally, IEM was used to confirm the presence of the anti-DMPO probe in plasma and nuclear membranes.

\section{Future directions}

The IST-mMRI approach can certainly be further applied to study free radicals associated longitudinally in oxidative stressrelated disease processes, as well as assess the effect of therapeutic agents that alter free radical levels. Mass spectrometry may need to be used to not only further assess whether the antiDMPO probe detected in heterogeneous tissue regions is essentially oxidized proteins (33) or oxidized lipids, or a combination of both, before the type of protein or lipid is identified. The current size of the probe may prohibit use in neurological studies with an intact BBB. The development of a smaller nanoparticlebased anti-DMPO probe, which may allow access through an intact $\mathrm{BBB}$, is currently being considered.

\section{References}

1. Barriga-González G, Olea-Azar C, Zuñiga-López MC, Folch-Cano C, Aguilera-Venegas B, Porcal W, González M, and Cerecetto H. Spin trapping: an essential tool for the study of diseases caused by oxidative stress. Curr Top Med Chem 15: 484-495, 2015.

2. Berg RMG, Moller K, and Bailey DM. Neuro-oxidativenitrosative stress in sepsis. J Cereb Blood Flow Metab 31: 1532-1544, 2011.

3. Berkowitz BA, Lenning J, Khetarpal N, Tran C, Wu JY, Berri AM, Dernay K, Haacke EM, Shafie-Khorassani F, Podolsky RH, Gant JC, Maimaiti S, Thibault O, Murphy GG, Bennett BM, and Roberts R. In vivo imaging of prodromal hippocampus CA1 subfield oxidative stress in models of Alzheimer disease and Angelman syndrome. FASEB $J$ 31: 4179-4186, 2017.

4. Berkowitz BA, Lewin AS, Biswal MR, Bredell BX, Davis $\mathrm{C}$, and Roberts R. MRI of retinal free radical production with laminar resolution in vivo. Invest Ophthamol Vis Sci 57: 577-585, 2016.

5. Berliner LJ. From spin-labeled proteins to in vivo EPR applications. Eur Biophys J 39: 579-588, 2010.

6. Berliner LJ, Khramtsov V, Fujii H, and Clanton TL. Unique in vivo applications of spin traps. Free Radic Biol Med 30: 489-499, 2011.

7. Bonini MG, Rota C, Tomasi A, and Mason RP. The oxidation of 2',7'-dichorofluorescin to reactive oxygen species: a self-fulfilling prophesy? Free Radic Biol Med 40: 968-975, 2006.

8. Bozza FA, D'Avila JC, Ritter C, Sonneville R, Sharshar T, and Dal-Pizzol F. Bioenergetics, mitochondrial dysfunction, and oxidative stress in the pathophysiology of septic encephalopathy. Shock 39: 10-16, 2013.

9. Buettner GR. Spin trapping: ESR parameters of spin adducts. Free Radic Biol Med 3: 259-303, 1987.

10. Coutinho de Souza P, Smith N, Atolagbe O, Ziegler J, Nijoku C, Lerner M, Ehrenshaft M, Mason RP, Meek B, Plafker SM, Saunders D, Mamedova N, and Towner RA. OKN-007 decreases free radicals levels in a preclinical F98 rat glioma model. Free Radical Biol Med 87: 157-168, 2015.

11. Davies MJ. Detection and characterisation of radicals using electron paramagnetic resonance (EPR) spin trapping and related methods. Methods 109: 21-31, 2016.

12. Davies MJ and Slater TF. The use of electron-spinresonance techniques to detect free-radical formation and tissue damage. Proc Nutr Soc 47: 397-405, 1988.

13. Du L, Huang S, Zhuang Q, Jia H, Rockenbauer A, Liu Y, Liu KJ, and Liu Y. Highly sensitive free radical detection by nitrone-functionalized gold nanoparticles. Nanoscale 6: 1646-1652, 2014.

14. Eto H, Hyodo F, Kosem N, Kobayashi R, Yasukawa K, Nakao M, Kiniwa M, and Utsumi H. Redox imaging of skeletal muscle using in vivo DNP-MRI and its application to an animal model of local inflammation. Free Radic Biol Med 89: 1097-1104, 2015.

15. Fakhruddin S, Alanzi W, and Jackson KE. Diabetes-induced reactive oxygen species: mechanism of their generation and role in renal injury. J Diabetes Res 2017: 8379327, 2017.

16. Gomez-Mejiba SE, Zhai Z, Akram H, Deterding LJ, Hensley K, Smith N, Towner RA, Tomer KB, Mason RP, and Ramirez DC. Immuno-spin trapping of protein and DNA radicals: "tagging" free radicals to locate and understand the redox process. Free Radic Biol Med 46: 853-865, 2009. 
17. Gomez-Mejiba SE, Zhai Z, Della-Vedova MC, Muñoz MD, Chatterjee S, Towner RA, Hensley K, Floyd RA, Mason $\mathrm{RP}$, and Ramirez DC. Immuno-spin trapping from biochemistry to medicine: advances, challenges, and pitfalls. Focus on protein-centered radicals. Biochim Biophys Acta 1840: 722-729, 2014.

18. Gupta N, Reja SI, Bhalla V, Gupta M, Kaur G, and Kumar M. An approach for the selective detection of nitric oxide in biological systems: an in vitro and in vivo perspective. Chem Asian J 11: 1020-1027, 2016.

19. Hawkins CL and Davies MJ. Detection and characterisation of radicals in biological materials using EPR methodology. Biochim Biophys Acta 1840: 708-721, 2014.

20. Hu JJ, Wong NK, Ye S, Chen X, Lu MY, Zhao AQ, Guo Y, Ma AC, Leung AY, Shen J, and Yang D. Fluorescent probe HKSOX-1 for imaging and detection of endogenous superoxide in live cells and in vivo. J Am Chem Soc 137: 6837-6843, 2015.

21. Huang Y, Yu F, Wang J, and Chen L. Near-infrared fluorescence probe for in situ detection of superoxide anion and hydrogen polysulfides in mitochondrial oxidative stress. Anal Chem 88: 4122-4129, 2016.

22. Hyodo F, Ito S, Eto H, Nakaji T, Yasukawa K, Kobayashi $\mathrm{R}$, and Utsumi H. Development of redox metabolic imaging using endogenous molecules. Yakugaku Zasshi 136: 11071114, 2016.

23. Ichikawa $\mathrm{K}$ and Yasukawa $\mathrm{K}$. Imaging in vivo redox status in high spatial resolution with OMRI. Free Radic Res 46: 1004-1010, 2012.

24. Iverson NM, Barone PW, Shandell M, Trudel LJ, Sen S, Sen F, Ivanov V, Atolia E, Farias E, McNicholas TP, Reuel N, Parry NM, Wogan GN, and Strano MS. In vivo biosensing via tissue-localizable near-infrared-fluorecent single-walled carbon nanotubes. Nat Nanotechnol 8: 873-880, 2013.

25. Iverson NM, Strano MS, and Wogan GN. In vivo delivery of nitric oxide-sensing, single-walled carbon nanotubes. Curr Protoc Chem Biol 7: 93-102, 2015.

26. Janzen EG. Spin trapping. Methods Enzymol 104: 188-198, 1984.

27. Janzen EG, Poyer JL, Schaefer CF, Downs PE, and DuBose CM. Biological spin trapping. II. Toxicity of nitrone spin traps: dose-ranging in the rat. $J$ Biochem Biophys Methods 30: 239-247, 1995.

28. Janzen EG, Stronks HJ, Dubose CM, Poyer JL, and McCay PB. Chemistry and biology of spin-trapping radicals associated with halocarbon metabolism in vitro and in vivo. Environ Health Perspect 64: 151-170, 1985.

29. Janzen EG and Towner RA. Aminoxyl radicals as MRI contrast agents. In: Bioactive Spin Labels, edited by Zhdanov R. Berlin: Springer-Verlag, 1992, pp. 573-583.

30. Jerome NP, Papoutsaki MV, Orton MR, Parkes HG, Winfield JM, Boss MA, Leach MO, deSouza NM, and Collins DJ. Development of a temperature-controlled phantom for magnetic resonance quality assurance of diffusion, dynamic, and relaxometry measurements. Med Phys 43: 2998, 2016.

31. Khoo NK, Cantu-Medellin N, St Croix C, and Kelley EE. In vivo immuno-spin trapping: imaging the footprints of oxidative stress. Curr Protoc Cytom 74: 12.42.1-11, 2015.

32. Kumara Dhas M, Utsumi H, Jawahar A, and Milton Franklin Benial A. Dynamic nuclear polarization properties of nitroxyl radical in high viscous liquid using Overhauserenhanced magnetic resonance imaging (OMRI). J Magn Reson 257: 32-38, 2015.
33. Lardinos OM, Detweiler CD, Tomer KB, Mason RP, and Deterding LJ. Identifying the site of spin trapping in proteins by a combination of liquid chromatography, ELISA, and off-line tandem mass spectrometry. Free Radic Biol Med 44: 893-906, 2008.

34. Li Z, Liang T, Lv S, Zhuang Q, and Liu Z. A rationally designed upconversion nanoprobe for in vivo detection of hydroxyl radical. J Am Chem Soc 137: 11179-11185, 2015.

35. Liu F, Du J, Song D, Xu M, and Sun G. A sensitive fluorescent sensor for the detection of endogenous hydroxyl radicals in living cells and bacteria and direct imaging with respect to its ecotoxicity in living zebra fish. Chem Comm 52: 4636-4639, 2016.

36. Liu X, Liu S, and Liang G. Fluorescence turn-on for the highly selective detection of nitric oxide in vitro and in living cells. Analyst 141: 2600-2605, 2016.

37. Mason RP. Assay of in situ radicals by electron spin resonance. Methods Enzymol 105: 416-422, 1984.

38. Mason RP. In vivo spin trapping-from chemistry to toxicology. In: Toxicology of the Human Environment: The Critical Role of Free Radicals, edited by Rhodes C. London: Taylor and Francis, 2000, pp. 49-70.

39. Mason RP. Using anti-5,5-dimethyl-1-pyrroline N-oxide (anti-DMPO) to detect protein radicals in time and space with immuno-spin trapping. Free Radic Biol Med 36: 1214-1223, 2004.

40. Mason RP. Imaging free radicals in organelles, cells, tissue, and in vivo with immuno-spin trapping. Redox Biol 8: 422429, 2016

41. McCay PB. Application of ESR spectroscopy in toxicology. Arch Toxicol 60: 133-137, 1987.

42. Newsholme P, Cruzat VF, Keane KN, Carlessi R, and Homen de Bittencourt Jr PI. Molecular Mechanisms of ROS production and oxidative stress in diabetes. Biochem $J$ 473: 4527-4550, 2016.

43. Niedzielska E, Smaga I, Gawlik M, Moniczewski A, Stankowicz P, Pera J, and Filip M. Oxidative stress in neurodegenerative diseases. Mol Neurobiol 53: 4094-4125, 2016.

44. Ramirez DC and Mason RP. Immuno-spin trapping: detection of protein-centered radicals. Curr Protoc Toxicol 17: 17, 2005.

45. Rayner CL, Gole GA, Bottle SE, and Barnett NL. Dynamic, in vivo, real-time detection of retinal oxidative status in a model of elevated intraocular pressure using a novel, reversibly responsive, profluorescent nitroxide probe. Exp Eye Res 129: 48-56, 2014.

46. Ribou AC. Synthetic sensors for reactive oxygen species detection and quantification: a critical review of current methods. Antioxid Redox Signal 25: 520-533, 2016.

47. Rinaldi M, Caffo M, Minutoli L, Marini H, Abbritti RV, Squadrito F, Trichilo V, Valenti A, Barresi V, Altavilla D, Passalacqua M, and Caruso G. ROS and brain gliomas: an overview of potential and innovative therapeutic strategies. Int J Mol Sci 17: Pii: E984, 2016.

48. Shvedova AA, Kisin ER, Murray AR, Mouithys-Mickalad A, Stadler K, Mason RP, and Kadiiska M. ESR evidence for in vivo formation of free radicals in tissue of mice exposed to single-walled carbon nanotubes. Free Radic Biol Med 73: 154-165, 2014.

49. Sorce S, Stocker R, Seredenina T, Holmdahl R, Aguzzi A, Chio A, Depaulis A, Heitz F, Olosson P, Olsson T, Duveau V, Sanoudou D, Skosgater S, Vlahou A, Wasquel D, Krause KH, and Jaquet V. NADPH oxidases as drug targets 
and biomarkers in neurodegenerative diseases: what is the evidence? Free Radic Biol Med 112: 387-396, 2017.

50. Spear JT and Gore JC. New insights into rotating frame relaxation at high field. NMR Biomed 29: 1258-1273, 2016.

51. Tain RW, Scotti AM, Li W, Zhou XJ, and Cai K. Imaging short-lived reactive oxygen species (ROS) with endogenous contrast MRI. J Magn Reson Imaging 47: 222-229, 2018.

52. Togashi H, Aoyama M, and Oikawa K. Imaging of reactive oxygen species generated in vivo. Magn Reson Med 75: 1375-1379, 2016.

53. Towner RA. Chemistry of spin trapping. In: Toxicology of the Human Environment: The Critical Role of Free Radicals, edited by Rhodes C. London: Taylor and Francis, 2000, pp. 7-24.

54. Towner RA, Garteiser P, Bozza F, Smith N, Saunders D, d'Avila JCP, Magno F, Oliveira MF, Ehrenshaft M, Lupu F, Silasi-Mansat R, Ramirez DC, Gomez-Mejiba SE, Mason $\mathrm{RP}$, and Faria-Neto HCC. In vivo detection of free radicals in mouse septic encephalopathy using molecular MRI and immuno-spin-trapping. Free Radic Biol Med 65: 828-837, 2013.

55. Towner RA, Smith N, Saunders D, Carrizales J, Lupu F, Silasi-Mansat R, Ehrenshaft $\mathrm{M}$, and Mason RP. In vivo targeted molecular magnetic resonance imaging of free radicals in diabetic cardiomyopathy within mice. Free Radic Res 49: 1140-1146, 2015.

56. Towner RA, Smith N, Saunders D, De Souza PC, Henry L, Lupu F, Silasi-Mansat R, Ehrenshaft M, Mason RP, Gomez-Mejiba SE, and Ramirez DC. Combined molecular MRI and immuno-spin-trapping for in vivo detection of free radicals in orthotopic mouse GL261 gliomas. Biochim Biophys Acta 1832: 2153-2161, 2013.

57. Towner RA, Smith N, Saunders D, Henderson M, Downum K, Lupu F, Silasi-Mansat R, Ramirez DC, Gomez-Mejiba $\mathrm{SE}$, Bonini MG, Ehrenshaft $\mathrm{M}$, and Mason RP. In vivo imaging of immuno-spin trapped radicals with molecular MRI in a mouse diabetes model. Diabetes 61: 2405-2413, 2012.

58. Towner RA, Smith N, Saunders D, Lupu F, Silasi-Mansat R, West M, Ramirez DC, Gomez-Mejiba SE, Bonini MG, Mason RP, Ehrenshaft M, and Hensley K. In vivo detection of free radicals using molecular MRI and immuno-spintrapping in a mouse model for amyotrophic lateral sclerosis (ALS). Free Radic Biol Med 63: 351-360, 2013.

59. Utsumi $\mathrm{H}$ and Hyodo $\mathrm{F}$. Free radical imaging using in vivo dynamic nuclear polarization-MRI. Methods Enzymol 564: 553-571, 2015.

60. Villamena FA and Zweier JL. Detection of reactive oxygen and nitrogen species by EPR spin trapping. Antioxid Redox Signal 6: 619-629, 2004.

61. Yamamoto S, Watabe T, Ikeda H, Ichikawa K, Nakao M, Kato K, and Hatazawa J. Development of a PET/OMRI combined system for simultaneous imaging of positron and free radical probes for small animals. Med Phys 43: 5676, 2016.

62. Zhang J, Li C, Zhang R, Zhang F, Liu W, Liu X, Le SM-Y, and Zhang H. A phosphinate-based near-infrared fluores- cence probe for imaging the superoxide radical anion in vitro and in vivo. Chem Commun 52: 2679-2682, 2016.

Address correspondence to: Dr. Rheal A. Towner Advanced Magnetic Resonance Center Oklahoma Medical Research Foundation $825 \mathrm{NE}$ 13th Street Oklahoma City, OK 73104

E-mail: rheal-towner@omrf.org

Date of first submission to ARS Central, October 16, 2017; date of acceptance, October 28, 2017.

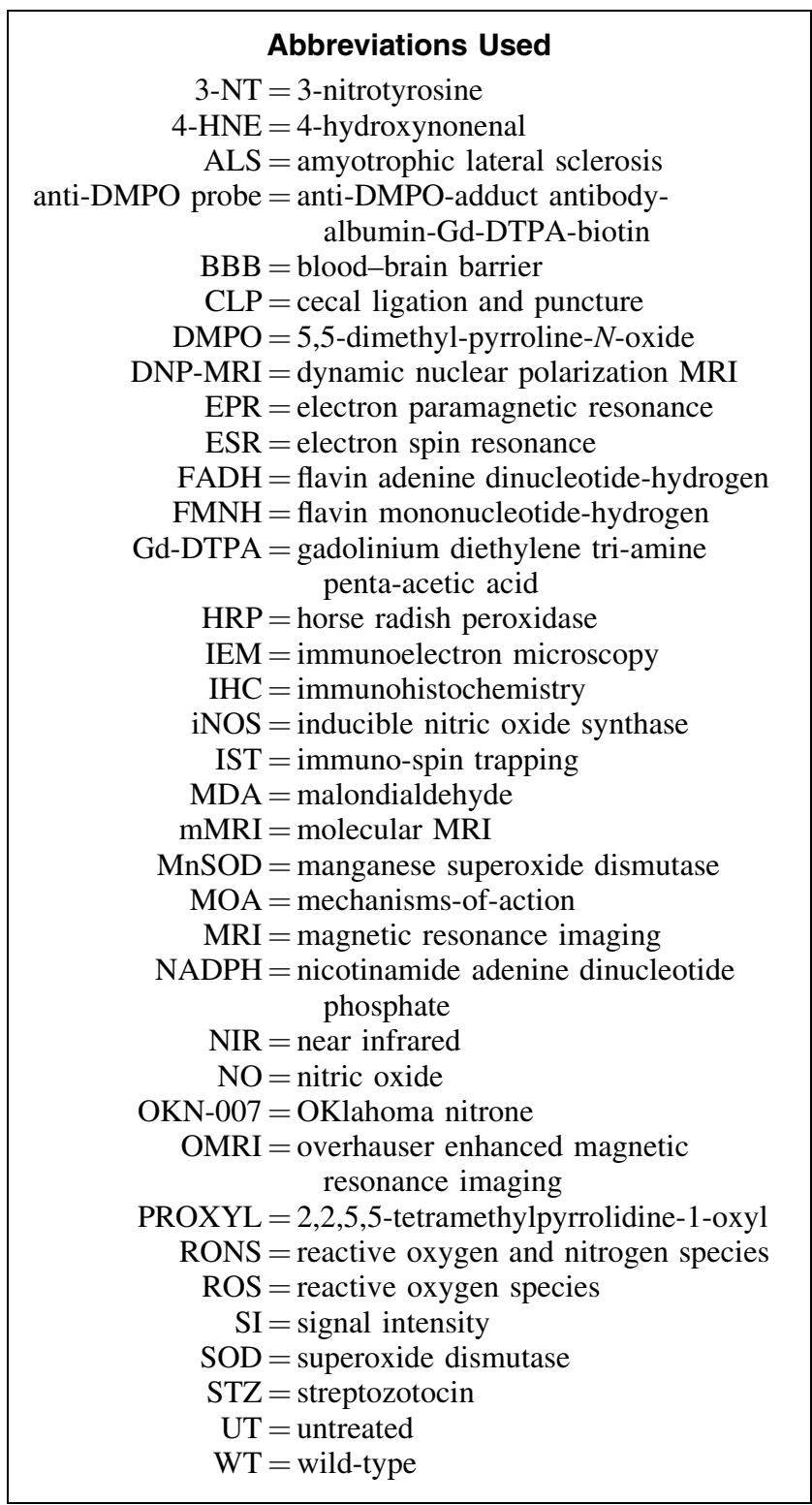

